\title{
Evaporation and Combustion of a Drop of Liquid Fuel-A Review
}

\section{Dabilgou Téré1,2*, Tubreoumya Guy Christian1, Haro Kayaba3, Bagre Boubou1, Sandwidi Sayouba1, Daho Tizane1, Koulidiati Jean', Sanogo Oumar³, Zeghmati Belkacem², Bere Antoine ${ }^{1}$}

\author{
${ }^{1}$ Laboratoire de Physique et de Chimie de l'Environnement (LPCE), Université Joseph Ki-Zerbo, \\ Ouagadougou, Burkina Faso \\ ${ }^{2}$ Laboratoire de Mathématiques et Physiques (LAMPS), Université de Perpignan via Domitia, \\ Perpignan, France \\ ${ }^{3}$ Centre National de la Recherche Scientifique et Technologique (CNRST), Institut de Recherche en Sciences Appliquées et \\ Technologies (IRSAT), Ouagadougou, Burkina Faso \\ Email: *terejdabilgou@yahoo.fr
}

How to cite this paper: Téré, D., Christian, T.G., Kayaba, H., Boubou, B., Sayouba, S., Tizane, D., Jean, K., Oumar, S., Belkacem, Z. and Antoine, B. (2022) Evaporation and Combustion of a Drop of Liquid Fuel-A Review. Smart Grid and Renewable Energy, 13, 28-54

https://doi.org/10.4236/sgre.2022.132003

Received: November 15, 2021

Accepted: February 11, 2022

Published: February 14, 2022

Copyright (c) 2022 by author(s) and Scientific Research Publishing Inc. This work is licensed under the Creative Commons Attribution International License (CC BY 4.0).

http://creativecommons.org/licenses/by/4.0/

\begin{abstract}
The accelerated depletion of oil reserves and the often exorbitant cost of fossil fuels contribute to the development of fuels from renewable sources. The objective of this work is to analyze the influence of the properties of renewable fuels on their evaporation in natural convection, their combustion and their use in internal combustion engines. A summary of the various numerical and experimental works from the literature has been presented in this work. This work focuses on the numerical modelling of the natural convection evaporation of an isolated drop of a liquid fuel in natural convection. The transfers in the liquid and vapour phases are described by the conservation equations of mass and species, momentum and energy. The main feature of this work is the consideration of advection, azimuthal angle and thickness of the vapour phase of the drop during evaporation of the drop.
\end{abstract}

\section{Keywords}

Drop, Evaporation, Combustion, Convection, Modelling, Temperature

\section{Introduction}

The numerical approach to the problems of evaporation and combustion of fuel droplets has experienced a considerable growth and interest of researchers in recent years [1] [2] [3]. This new approach, for the most part, takes into account the heat and mass transfer processes in the liquid and vapour phases as well as 
the coupling between them [4] [5] [6] [7] [8]. Therefore, it is important to have a broad understanding of the physical phenomena and mechanisms involved in the evaporation and combustion of fuel droplets in diesel engines, especially in the design and optimization of combustion chambers. In view of the complexity of nature to study the phenomena, the evaporation of an isolated drop in natural convection is often studied and the spherically symmetrical model is representative [4] [9] [10] [11].

Indeed, fuel evaporation in internal combustion engines has a strong influence on pollutant emissions, ignition delays and overall combustion efficiency and when it enters a high temperature environment its evaporation and combustion provides energy for propulsion [9] [12] [13]. However, the two-phase flow phenomenon, existing in the evaporation of a drop of liquid fuel, remains a very important area for researchers to explore. Despite the growth and development of numerical techniques, difficulties with the two-phase flow phenomenon still exist and research continues to address them. Thus, numerical techniques have been undertaken to solve this problem [2] [8] [14]-[19]. These methods are mainly oriented by following the time evolution of the radius squared regression and the evolution or transport of the interface i.e. the surface temperature [20].

Also, during the fuel spray, interactions between the fuel droplets occur which produce different physical mechanisms and make the evaporation phenomenon complex [12] [21] [22] [23]. Moreover, it is difficult to isolate and study these physical mechanisms individually [7] [15] [24]. Indeed, fuel, injected as a liquid spray, usually burns as a group rather than individually in the diesel engine cylinder. Nevertheless, it appears that the study of the evaporation of an isolated drop is generally carried out and gives a good first approximation of the evaporation phenomenon [10] [25] [26]. Thus, several authors have been interested in the phenomenon of evaporation of isolated drops, in particular the heat and mass transfers that link the liquid and gaseous phases as well as the coupling between these two phases [4] [27] [28]. The interest, under these conditions of evaporation of isolated droplets of liquid fuel, was thus mainly focused on the determination of the fundamental processes of heat and mass transfer. Despite the existence of many evaporation models in the literature, most modelling studies do not take into account advection, which is however present in the evaporation phenomenon of drops. In addition, not all evaporation models take into account the azimuth angle, yet in real conditions, evaporation is accompanied by a variation in azimuth angle. Also, some studies neglect the thickness of the gaseous phase, which makes it possible to follow the evolution of the evaporation of the drop and the heat and mass transfer phenomena. Moreover, the evaporation of an isolated drop in an ambient medium is driven by a relative motion of the external fluid and is subject to a set of forces. It's therefore important to control the nature of the flow (laminar or turbulent), the evolution of the physicochemical properties of the biofuel (biodiesel and heptane in this case) and the surrounding environment. It is also necessary to follow the evolution and the influence of the thermal and mass exchanges between the two phases, the influence 
of the possible interactions between the particles and especially the effect that they generate on the variation of the physical properties of the flow (drag force, relative speed, ...).

In addition, it has been shown that the phenomenon of natural convection can considerably modify the rates of heat and mass transfer between the evaporating fuel drops and the surrounding gas phase. Conditions in a combustion chamber can potentially promote and reduce natural convection due to the high pressure environment and small drop size [2] [29] [30]. In this case, this phenomenon contributes to the increase of energy and mass transfers between the evaporating drop and the surrounding gas. This means that under ambient conditions, the integration of these effects could become essential in the determination of the evaporation phenomena of drops. Therefore, in this work, the phenomena of natural convection, advection and azimuthal angle are taken into account.

The modelling of evaporation in natural convection of an isolated fuel drop has therefore been carried out.

This work therefore focuses on a review of the current literature on the vaporization and combustion of an isolated drop of fuel. The main axes developed in this work are oriented towards the general description of the vaporization phenomena and the different vaporization mechanisms. Secondly, the influence of different parameters on vaporization such as convection (forced or natural), temperature and pressure has been evaluated. Numerical and experimental studies highlighting the different models and materials used in the literature and their limitations are mentioned in this manuscript. Finally, a critical look at the combustion literature and a global synthesis complete this work.

\section{General Description of Evaporation Phenomena}

\subsection{Phenomena Involved in Injection, Evaporation and Combustion}

In the combustion chambers of an internal combustion engine, fuel is injected in the form of a mist of liquid drops $<$ sprays $>$ as fine as possible. During the injection of this liquid fuel, several phenomena take place: disintegration of the liquid, atomization, turbulent dispersion, etc. During this stage, which precedes combustion, heat and mass transfers between the drops and their gaseous environment come into play. The phenomena are extremely numerous and varied, making the study of the whole very complex [7] [9] [31] [32]. Simple experiments are then defined to isolate each of the above mentioned phenomena in order to model them. The numerical simulation of dense two-phase flows in an internal combustion chamber of a diesel engine must take into account the modeling of several rather complex physical processes.

Among these phenomena, the modeling of drop evaporation allows to quantify the amount of fuel vapor supplied to the gas phase in order to simulate the spray combustion. In order to keep calculation times reasonable, the fuel is al- 
ways represented by a liquid composed of a single species (heptane, n-octane, $\mathrm{n}$-decane for paraffin or iso-octane for petrol). However, these fuels are actually composed of several hundred components, all with different physicochemical characteristics [25] [33] [34] [35]. One of the most important properties is volatility, which varies considerably from one species to another, and the different components of a fuel can thus cover a wide range of boiling temperatures, above a hundred degrees [36] [37] [38] [39]. This affects the dynamics of evaporation as some species vaporize faster than others [39] [40]. The composition of the drop changes throughout its trajectory in the combustion chamber where the vapour is not uniform. The different species react differently and combustion phenomena such as ignition, flame propagation, flame stability or pollutant emissions can be affected [41].

\subsection{Drop Evaporation in a Stagnant Medium}

The evaporation of a drop of fuel remains one of the most important processes of combustion in a diesel engine combustion chamber [4]. It depends on the temperature of the drop and the temperature of the vapour phase as well as on the vapour pressure [42] [43] [44]. Consider a single-component, isolated and immobile drop initially placed in a gaseous medium. At the initial moment, the temperature at the surface of the liquid $\left(\mathrm{T}_{\mathrm{S}}\right)$ is lower than the temperature of the surrounding medium, the vapour pressure at its surface is lower than the pressure of the gaseous phase [9] [12]. Two phenomena occur:

- A quantity of heat is transferred by conduction-convection from the surrounding medium to the surface of the drop causing an increase in the temperature of the drop and the vapour concentration at its surface,

- Vapour concentration gradients between the surface of the drop and the surrounding medium cause evaporation of the liquid phase by particle diffusion.

If the temperature of the drop increases in the course of time, the evaporation process of the drop continues until it reaches self-ignition. Figure 1 illustrates the phenomena of drop evaporation.

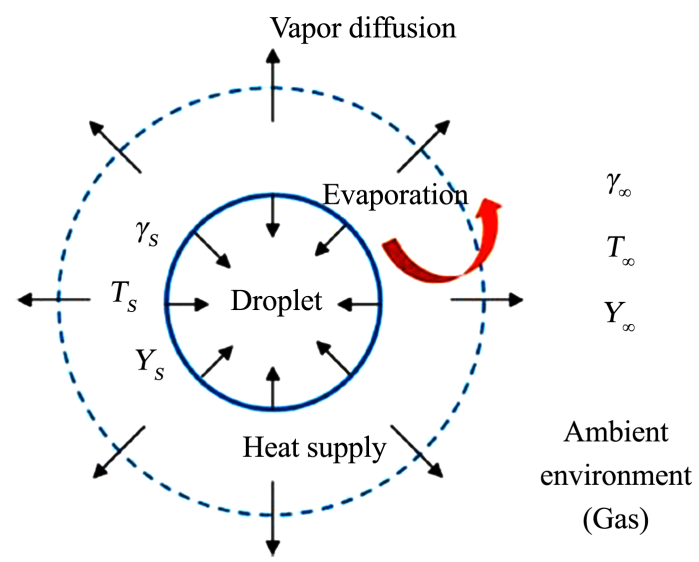

Figure 1. Physical phenomenon of a droplet evaporation in a stagnant medium [45]. 


\section{Evaporation Mechanisms}

The mechanisms of evaporation of a drop are:

- Heat transfers: related to temperature gradients,

- Quantity of movements transferred: induced by the gas flow and materializing in the form of a boundary layer,

- Mass transferred: caused by the concentration gradient through evaporation. Both diffusive and convective.

To understand the mechanisms of jet evaporation, it is essential to decouple the problem. On the one hand, there are heat exchange mechanisms that directly participate in the evaporation of the drop and on the other hand, there are dynamic effects (atomization) that indirectly participate in this atomization [45].

The formation of a large number of droplets by atomization increases the exchange surface of the droplets, which promotes heat transfer and thus evaporation [3] [9] [13].

The consideration of convection in the evaporation of a drop is very important because the viscous friction generated by the relative movement of the drop causes a decrease in the mass fraction of vapour at its surface [37] [46]. Convection avoids the phenomenon of vapour accumulation and on the other hand it accelerates the evaporation of the drop and decreases its life span. The coupling of these different types of transfers is shown by their dependence on common physical quantities linked to the properties of the different media, thermodynamic variables and the thermophysical properties of the fuel [25] [33] [35].

\section{Influence of Different Parameters on the Vaporization of Drops}

\subsection{Influence of Convection}

The influence of convection on the evaporation of a drop has been the subject of numerous works. Thus, Renksizbulut et al. in 1983, Scherrer in 1987 [47] [48] were interested in the phenomena of convection on evaporation. These authors describe the influence of the speed of the vapour phase flow around the drop during evaporation. It is also shown that the flow can modify the duration of vaporization. There is little work on the evaporation of one or more spherical drops in natural convection. However, it is worth noting that some work on evaporation in natural convection such as that of Ranz and Marshall which led to the development of correlation of Nusselt and Sherwood numbers validated by theoretical models and used in several works.

Yuge proposed a semi-empirical expression for the Nusselt numbers as a function of the thermal Grashof number based on a thermocouple measurement of the fuel drop temperature [11].

Experimental results on forced convection have shown mixed results (Table 1 and Table 2) [4] on the evaporation or combustion of one or more drops, which are summarized in the following tables. 
Table 1. Main results of evaporation and combustion of a fuel drop in natural convection [4].

\begin{tabular}{|c|c|c|c|}
\hline AUTHORS & STUDY SYSTEM & METHOD & RESULTS \\
\hline Ranz and Marshall (1952) & $\begin{array}{l}\text { Spherical drop } \\
\text { in evaporation }\end{array}$ & $\begin{array}{l}\text { Experimental: } \\
\text { thermocouple measurement }\end{array}$ & $\begin{array}{l}\overline{N u}=2+0.6 \cdot G r^{1 / 4} \cdot \operatorname{Pr}^{1 / 3} \\
\overline{S h}=2+0.6 \cdot G r^{1 / 4} \cdot S c^{1 / 3} \\
G r, P r \text { et } S c \text { no defined }\end{array}$ \\
\hline Yuge (1960) & $\begin{array}{l}\text { Evaporating } \\
\text { spherical drop }\end{array}$ & $\begin{array}{l}\text { Experimental: } \\
\text { thermocouple measurement }\end{array}$ & $\begin{array}{l}\overline{N u}=2+0.392 \cdot G r^{0.25} \\
1 \leq G r \leq 10^{5}\end{array}$ \\
\hline Amoto and Tien (1972) & $\begin{array}{l}\text { Spherical drop } \\
\text { in evaporation }\end{array}$ & $\begin{array}{c}\text { Experimental: temperature } \\
\text { measurement by thermocouple }\end{array}$ & $\begin{array}{l}3 \times 10^{5} \leq G r_{T} \cdot \operatorname{Pr} \leq 8 \times 10^{8} \\
\overline{N u}=2+C \cdot(G r \cdot P r)^{0.25} \\
C=0.05 \pm 0.009\end{array}$ \\
\hline $\begin{array}{l}\text { Sato and Mitsuhirotsue, } \\
\text { Niwa (1990) }\end{array}$ & $\begin{array}{l}\text { Spherical drop } \\
\text { in combustion }\end{array}$ & $\begin{array}{l}\quad \text { Experimental: } \\
\text { - } \quad \text { High pressure chamber } \\
\text { - } \quad \text { CCD camera }\end{array}$ & Ranz and Marshall correlations \\
\hline Daïf and al (199) & $\begin{array}{l}\text { Spherical drop } \\
\text { in evaporation }\end{array}$ & $\begin{array}{c}\text { Numerical: } \\
\text { boundary layer model }\end{array}$ & $\begin{array}{l}\overline{N u}=2+0.59 \cdot\left(1+B_{T i}\right)^{-0.59} \overline{G r}^{-1 / 4} \cdot \operatorname{Pr}^{1 / 3} \\
\overline{S h}=2+0.57 \cdot\left(1+B_{M i}\right)^{0.09} G^{-1 / 4} \cdot S c^{1 / 3} \\
4 \times 10^{-5} \leq \overline{G r} \leq 7.7 \times 10^{4} \\
0.67 \leq \operatorname{Pr} \leq 0.91 ; \quad 0 \leq B_{T} \leq 2.6 \\
0.9 \leq S c \leq 3, \quad 0 \leq B_{M} \leq 2.44\end{array}$ \\
\hline
\end{tabular}

Table 2. Main results of evaporation and forced convection combustion of a fuel drop [4].

\begin{tabular}{|c|c|c|c|}
\hline AUTHORS & STUDY SYSTEM & METHOD & RESULTS \\
\hline Fuchs (1959) & $\begin{array}{l}\text { Spherical drop } \\
\text { in evaporation }\end{array}$ & $\begin{array}{l}\text { Experimental: } \\
\text { thermocouple }\end{array}$ & $\begin{array}{l}\overline{N u}=2+0.6 \operatorname{Re}^{1 / 2} \cdot \operatorname{Pr}^{1 / 3} \\
\operatorname{Re} \leq 200\end{array}$ \\
\hline Ranz et Marshall (1952) & $\begin{array}{l}\text { Spherical drop } \\
\text { in evaporation }\end{array}$ & $\begin{array}{l}\text { Experimental: } \\
\text { thermocouple }\end{array}$ & $\begin{array}{l}\overline{N u}=2+0.6 \operatorname{Re}^{1 / 2} \cdot \operatorname{Pr}^{1 / 3} \\
R e \leq 200\end{array}$ \\
\hline Renksizbulut et Yuen (1983) & $\begin{array}{c}\text { Porous spherical } \\
\text { drop in evaporation }\end{array}$ & $\begin{array}{l}\text { Numerical: finite } \\
\text { difference method }\end{array}$ & $\begin{array}{l}\overline{N u}(1+B)^{0.7}=2+0.9 R e^{0.57} \cdot \operatorname{Pr}^{1 / 3} \\
R e \leq 100\end{array}$ \\
\hline Renksizbulut et Yuen (1983) & Spherical drop in evaporation & $\begin{array}{l}\text { Experimental: } \\
\text { thermocouple }\end{array}$ & $\begin{array}{l}\overline{N u}(1+B)^{0.7}=2+0.57 \operatorname{Re}^{0.5} \cdot \operatorname{Pr}^{1 / 3} \\
0 \leq \operatorname{Re} \leq 150\end{array}$ \\
\hline Natarajan (1966) & Spherical droplet in combustion & $\begin{array}{l}\text { Experimental: } \\
\text { thermocouple }\end{array}$ & $\begin{array}{l}N u_{m}(1+B)=1.46 R e^{0.5} \\
\operatorname{Re} \leq 20\end{array}$ \\
\hline
\end{tabular}

These correlations developed by these authors for the average and local Nusselt and Sherwood numbers are used by others to quantify heat and mass transfer. However, some improvements are still needed to take into account several components in order to have a more detailed mechanism of the convective and conductive transfer phenomena arround the drops during its evaporation and combustion.

Dgheim et al., 2012 [27] developed new correlations for the Nusselt and Sher- 
wood numbers as a function of the Dgheim number. These common correlations for different multicomponent hydrocarbons are determined by the least squares method where the effect of Dgheim $(D G)$, Prandtl (Pr), Schmidt (Sc), mass $\left(B_{M}\right)$ and heat $\left(B_{T}\right)$. These Nusselt and Sherwood numbers have the following expressions

$$
\overline{N u}=2+2.1\left(1+B_{T}\right)^{0.68} \operatorname{Pr}^{0.5} \mathrm{DG}
$$

With $0.5 \prec D G \prec 5$ et $0.7 \prec P r \prec 0.86$

$$
\overline{S h}=2+1.78\left(1+B_{M}\right)^{-2} S c^{0.5} D G
$$

With $0.5 \prec D G \prec 5$ et $1.58 \prec S c \prec 3.13$

$$
\begin{gathered}
\overline{N u}=2+2.1\left(1+B_{T}\right)^{0.68} D G_{T} \\
\overline{S h}=2+1.78\left(1+B_{M}\right)^{-2} D G_{M}
\end{gathered}
$$

With $D G_{T}=D G \sqrt{P r}$ et $D G_{M}=D G \sqrt{S c}$

These Nusselt and Sherwood numbers as represented by Figure 2 and Figure 3.

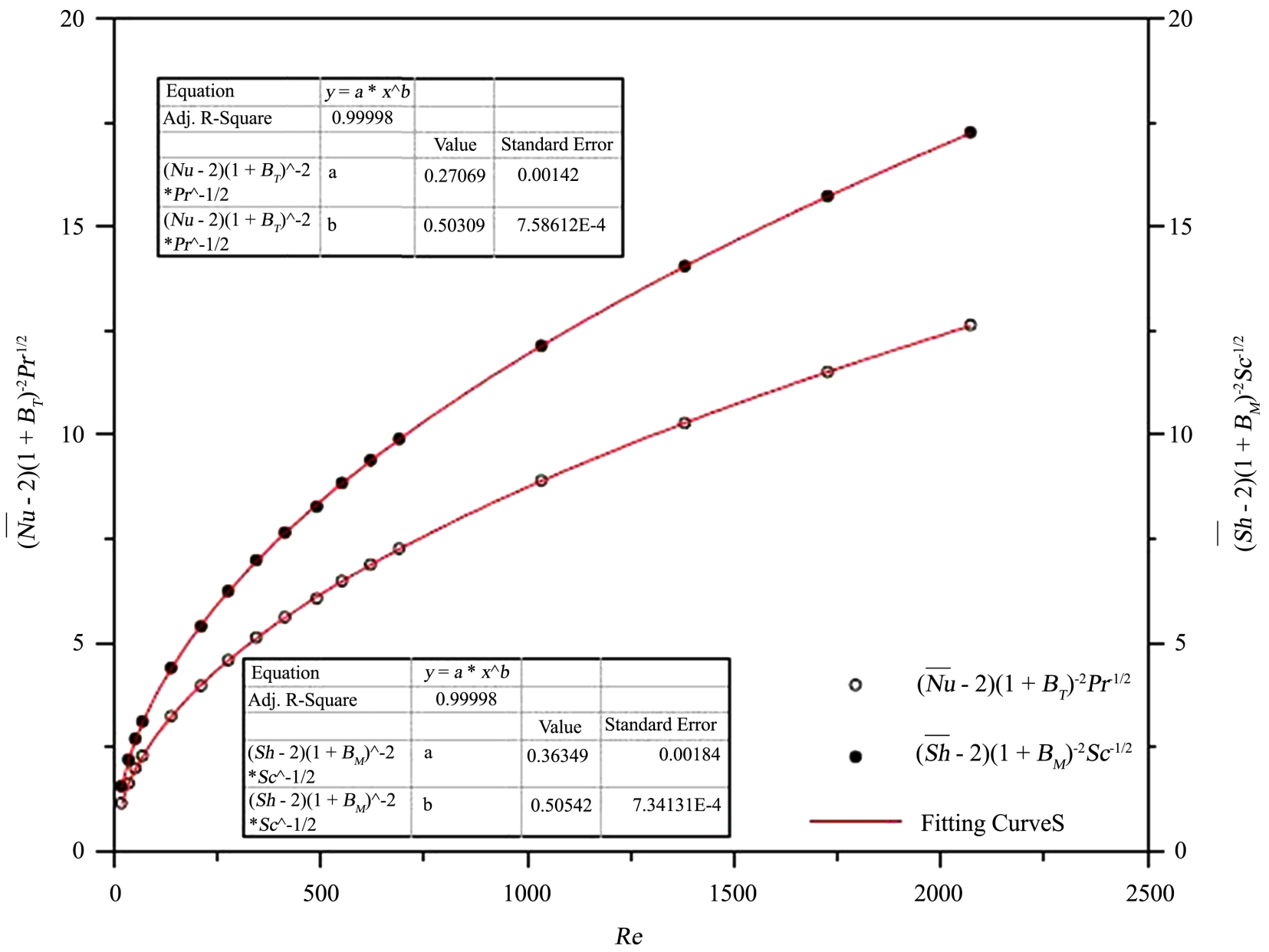

Figure 2. Correlations of the average Nusselt and Sherwood numbers as a function of the Reynolds, Prandtl, Schmidt, thermal and mass Spalding numbers [49]. 


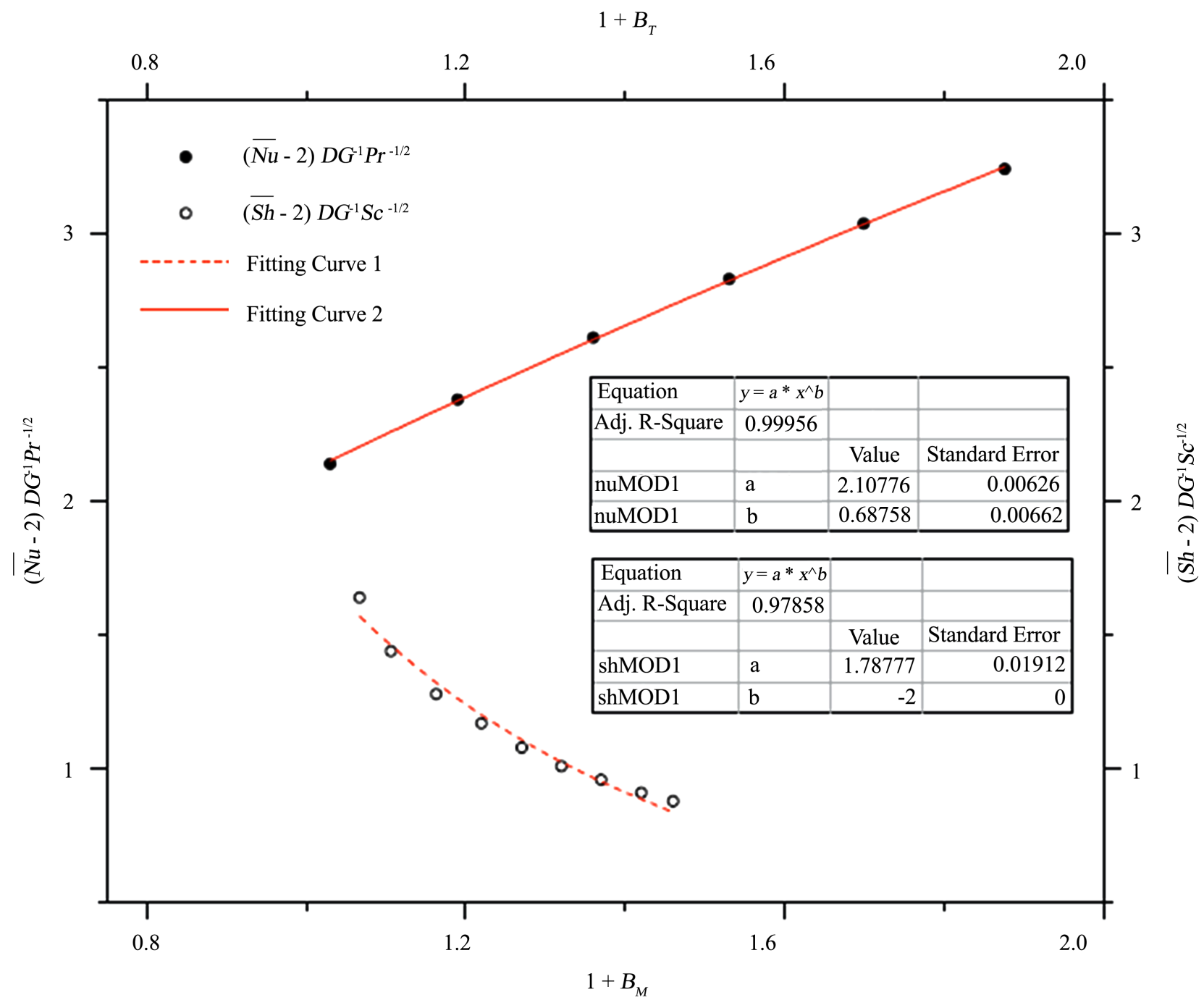

Figure 3. Correlations of the average Nusselt and Sherwood numbers as a function of the Dgheim, Prandtl, Schmidt, thermal and mass Spalding numbers [49].

These results are substantial, but do not show the link between mass concentration and the sensitive and local Nusselt and Sherwood numbers. However, in mass and heat transfer, concentration has a strong influence on evaporation phenomena.

\subsection{Influence of Natural Convection}

The symmetry of the problem can be broken when there are density gradients associated with mass and heat transfer in the vicinity of the drop. These gradients induce a non-radial motion of the gases around the drop generated by natural convection. The presence of non-radial convection accelerates the transfer rates and consequently reduces the lifetime of the drop. The lack of symmetry can be corrected by various semi-empirical correlations found in the literature. The most commonly used correlations are those of Rantz and Marshall (1952) [50]. 


\subsection{Influence of Forced Convection}

Several correlations have shown that the effects of forced convection on mass and heat transfer can be presented by a correlation coefficient as a function of Reynolds and Schmidth (or Prandtl) numbers, if the diffusion phenomenon controls the mass transfer between the liquid and gas phase, the correlation coefficient can be written as follows [18] [29] [49] [51]:

$$
k_{\text {corr }}=k_{\text {th }}\left(1+0.276 R e^{1 / 2} S c^{1 / 3}\right)
$$

$k_{t h}$ is the vaporization rate in the stagnant case.

In the case where heat transfer is the controlling mechanism, as in the case of combustion for example, this correction coefficient becomes:

$$
k_{\text {corr }}=k_{\text {th }}\left(1+0.276 \operatorname{Re}^{1 / 2} \operatorname{Pr}^{1 / 3}\right)
$$

In the case where heat transfer is the controlling mechanism, as in the case of combustion for example, this correction coefficient becomes:

The main function of convection is to accelerate the heat exchange process and the substance between the gas phase and the drop. Nevertheless, its consideration is very complex because the energy conservation equation has no analytical solution and is of importance even for a system as simple as a hard sphere subjected to a constant velocity flow. This is done by determining characteristic numbers that allow one to estimate the relative importance of the heat flow actually transmitted by convection compared to a purely conductive heat flow (Nusselt number, $\mathrm{Nu}$ ) or a purely diffusive material flow (Sherwood number, $S h)$. The $N u$ and $S h$ numbers are usually themselves expressed in terms of other numbers that characterize the gas flow (Reynolds number $R e$ ) and the transfer mechanisms (heat: Prandtl number $P r$, matter: Schmidt number $S c$ ).

\section{Numerical Model of Literature}

\subsection{Gas Phase}

\subsubsection{The $\mathrm{d}^{2}$ Law and Its Assumptions}

The $\mathrm{d}^{2}$ law describes the vaporization and combustion of a drop of fuel. To describe the evaporation mechanisms of an isolated stagnant drop consisting of a single component vaporizing in a stagnant medium, several models have been developed [8] [15] [52] [53]. The so-called standard or quasi-stationary model was developed by Godsave (1953), Spalding (1953), Goldsmith \& Penner (1954) and Wise et al. (1955) [54] [55]. This model is based on assumptions described by Williams (1973) [56], Faeth (1977) [6] and Law (1982) [3]. We briefly present these assumptions:

$\checkmark$ The isolated drop is assumed to be immobile in a gaseous medium. Convective transfers are neglected and the only heat and mass exchanges between the liquid and vapour phases take place by conduction and diffusion. This leads to a spherical symmetry of the drop. Thus, the transfers are assumed to be one-dimensional;

$\checkmark$ The drop can be assimilated to a pure body; 
$\checkmark$ The transfers take place at the same pressure. The regression velocity of the drop radius is small compared to the speed of sound in the surrounding gas;

$\checkmark$ The thermophysical properties of the drop in gas phase (heat capacity, thermal conductivity, diffusion coefficient) are constant. The Lewis number $(L e)$ of the surrounding medium is considered equal to 1 , i.e.:

$$
L e=\frac{S c}{P r}=\frac{\lambda}{\rho c_{p} D}=1
$$

with $\left\{\begin{array}{l}\text { Sc }: \text { Schmidt number } \\ \operatorname{Pr}: \text { Prandtl number } \\ D: \text { diffusion coefficient }\end{array}\right.$

$\checkmark$ The liquid-vapour equilibrium at the surface of the drop is instantaneous because the phase change between the liquid and its vapour is rapid (high vaporization rate)

$\checkmark$ The drop is considered in a quasi-stationary state: the phenomena of mass and heat transport and the heating time are neglected. Its temperature is considered constant and uniform. These results are obtained by the rule of Sparrow et al. in 1958 [57] called the "rule of thirds":

$$
\begin{aligned}
& T_{\text {ref }}=\frac{2}{3} T_{s}+\frac{1}{3} T_{\infty} \\
& Y_{\text {ref }}=\frac{2}{3} Y_{s}+\frac{1}{3} Y_{\infty}
\end{aligned}
$$

$\checkmark$ The effects of mass diffusion due to a temperature gradient (Soret effect) and the heat fluxes produced by a mass gradient (Dufour effect) are neglected,

$\checkmark$ Inertia effects in the gas phase, oxidation and decomposition phenomena in the gas phase are neglected,

These hypotheses, in addition to the boundary conditions, make it possible to obtain a law for monitoring the evolution over time of the diameter of the drop (d) or $d^{2}$ law, which is a function of time, its initial diameter $\left(d_{0}\right)$ and the rate of vaporization $(k)$ :

$$
d^{2}-d_{0}^{2}=-k t
$$

with $k=$ constant

\subsubsection{Standard Model of the $\mathrm{d}^{2}$ Law}

Considering the above assumptions and formulas, the conservation equations of mass, energy and species are written as:

$$
\frac{\mathrm{d} m}{\mathrm{~d} r}=0
$$

where

$$
m=4 \pi r_{s}^{2} \rho_{g a z} U_{r, s}
$$

and $r_{s}$ is the radius of the drop and $U_{r, s}$ is the radial velocity of the vapour at the surface. 


\section{Energy conservation equation}

$$
r^{2} \rho_{g a z} U_{r, s} C_{p g a z} \frac{\mathrm{d} T}{\mathrm{~d} r}=\frac{\mathrm{d}}{\mathrm{d} r}\left(r^{2} \lambda_{g a z} \frac{\mathrm{d} T}{\mathrm{~d} r}\right)
$$

\section{Conservation of species equation}

$$
\frac{\mathrm{d}}{\mathrm{d} r}\left[r^{2}\left(\rho_{g a z} U_{r, s} Y-\rho_{g a z} D_{g a z} \frac{\mathrm{d} Y}{\mathrm{~d} r}\right)\right]
$$

\section{Boundary conditions}

- At the surface of the drop and at infinity

$$
\begin{aligned}
& \left\{\begin{array}{l}
T\left(r=r_{s}\right)=T_{s} \\
T\left(r=r_{\infty}\right)=T_{\infty}
\end{array}\right. \\
& \left\{\begin{array}{l}
Y\left(r=r_{s}\right)=Y_{s} \\
Y\left(r=r_{\infty}\right)=Y_{\infty}
\end{array}\right.
\end{aligned}
$$

- At the liquid-vapour interface

$$
4 \pi r_{s}^{2} \rho_{g a z} D_{g a z} \frac{\mathrm{d} Y}{\mathrm{~d} r}=\dot{m}\left(1-Y_{s}\right)
$$

Solving these equations together with the boundary conditions leads to the following results:

$$
\begin{gathered}
T=\frac{\left(T_{s}-T_{\infty}\right)}{\exp \left(\frac{-\dot{m} C p_{g a z}}{4 \pi \lambda_{g a z} r_{s}}\right)-1}\left[\exp \left(\frac{-\dot{m} C p_{\text {gaz }}}{4 \pi \lambda_{g a z} r}\right)-1\right]+T_{\infty} \\
\dot{m}=4 \pi r_{s} \rho_{g a z} D_{g a z} \ln \left(1+B_{T}\right) \\
B_{T}=\frac{C p_{g a z}\left(T_{s}-T_{\infty}\right)}{L_{v}}
\end{gathered}
$$

The mass fraction of the vapour in the gas phase and the evaporated liquid flow rate are obtained from the solution of Equations (15) and (16) above and given by:

$$
\begin{gathered}
Y=\frac{Y_{s}}{\exp \left(\frac{-\dot{m}}{4 \pi \rho_{g a z} D_{g a z}}\right)}\left[\exp \left(\frac{-\dot{m}}{4 \pi \rho_{\text {gaz }} D_{\text {gaz }}}\right)-1\right] \\
\dot{m}=4 \pi r_{s} \rho_{\text {gaz }} D_{g a z} \ln \left(1+B_{M}\right) \\
B_{M}=\frac{Y_{s}-Y_{\infty}}{1-Y_{s}}
\end{gathered}
$$

According to the quasi-stationarity condition of the $d^{2}$ law and by assumption the Spalding mass and thermal numbers are equal, hence $B_{M}=B_{T}=B$. This results in:

$$
\dot{m}=4 \pi r_{s} \rho_{g a z} D_{g a z} \ln (1+B)
$$

For a spherical drop we have: 


$$
\begin{gathered}
m=\frac{4}{3} \pi r^{3} \rho_{\text {goutte }} \\
\dot{m}=4 \pi r^{2} \rho_{\text {goutte }} D_{\text {gaz }} \frac{\mathrm{d} r}{\mathrm{~d} t}
\end{gathered}
$$

Combining the expressions in Equations (22) to (26), we get $d^{2}-d_{0}^{2}=-k \cdot t$. This expression is called the law in $d^{2}$ with $d$ the diameter of the drop, do the initial diameter of the drop, $t$ the time and $k$ the rate of vaporization and is given by:

$$
k=8 \frac{\lambda_{\text {gaz }}}{C p_{\text {gaz }} \rho_{\text {goutte }}} \ln (1+B)
$$

Daho et al., 2012 [26] showed the evolution of the evaporation constants of the two fuels obtained by the quasi-stationary model and the Ranz and Marshall correlation (taking into account or not the influence of the transient phase) as a function of temperature. The observation of the results through the figures shows that the evaporation constants of the cottonseed oil increase more rapidly than those of the diesel fuel when the temperature increases. Numerical and experimental representations are shown in Figure 4 and Figure 5 for different fuels.

The quasi-transitory phenomenon reported by Daho et al., 2012 is very important in the case of fuel evaporation and combustion but does not explicitly describe the mechanisms related to evaporation and the influence of the surrounding environment such as advection or the initial drop radius size. However, drop size and advection have an effect on the injection, atomization and disintegration of fuel drops and consequently on the evaporation and combustion of these fuels.

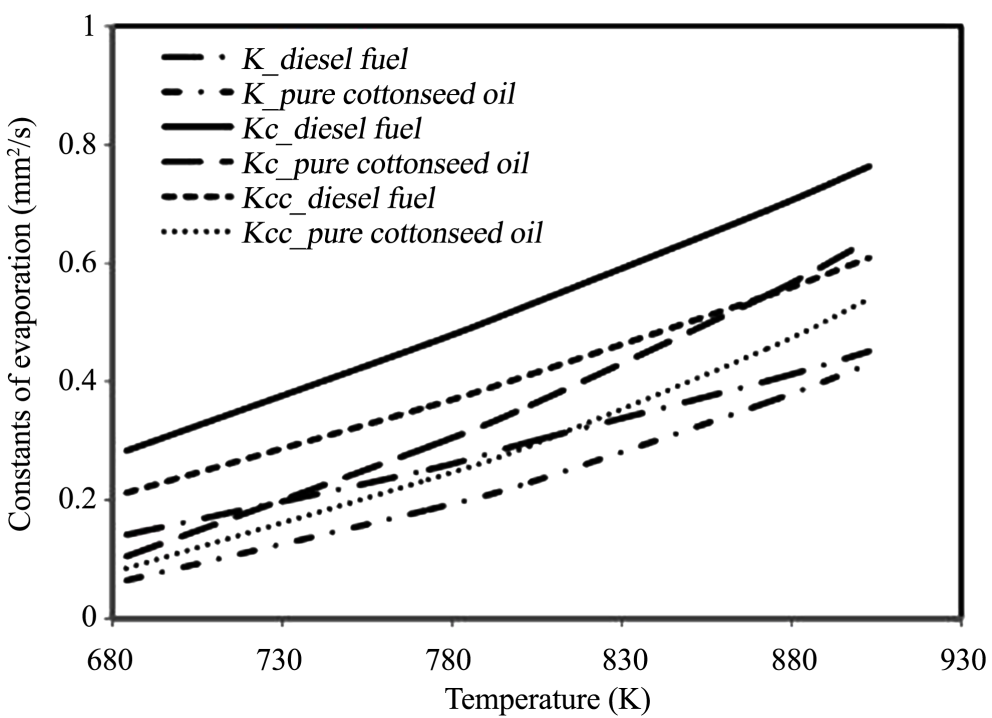

Figure 4. Theoretical constants of evaporation of diesel fuel and cottonseed oil. K, Kc and Kcc are respectively theoretical constants of evaporation from quasi-steady model, Ranz and Marshall correlation and Ranz and Marshall correlation taking into account the influence of heating phase of the droplet [26]. 


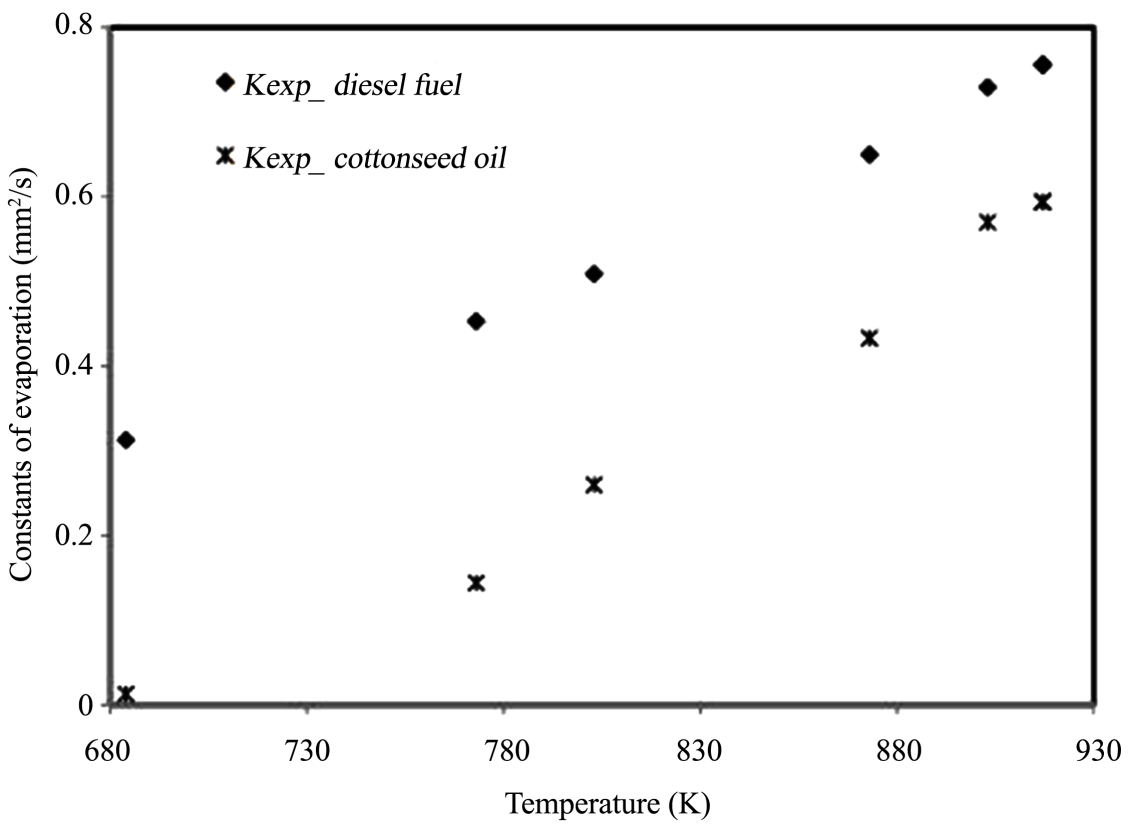

Figure 5. Experimental constants of evaporation of diesel fuel and cottonseed oil. Kexp_diesel fuel and Kexp_cottonseed oil are respectively the experimental constants of evaporation of diesel fuel and cottonseed oil [26].

$\mathrm{Gu}, \mathrm{Xin}, 2012$ [58] used the classical $\mathrm{d}^{2}$ law model to compare his experimental and numerical results with heptane and decane fuels. He finds satisfactory results with a slight deviation of less than $3 \%$. It should be remembered that in the case of natural convection evaporation, for most cases, only the time regression of the square of the diameter or the surface temperature remain the most easily observable parameters. The diameter squared regression and surface temperature versus time are summarised in a single figure and shown in Figure 6.

However, the work of G. Xin does not clearly present the experimental conditions and the error bars obtained. In addition, the difference in surface temperatures between the numerical model and the experimental one is not well justified. The influence of radiation in the case of evaporation should also be shown, as opposed to convection and conduction which are taken into account. Otherwise, it should be mentioned in the simplifying assumptions.

\subsection{Inadequacy of the $d^{2}$ Law}

The $\mathrm{d}^{2}$ law model has been verified in the literature by experimental results [6] [29] [30] (Faeth (1977); Chauveau (1990); Chesneau (1994)). However, it is not immune to many criticisms. In this paragraph, we will outline some of its shortcomings, which we present below:

$\checkmark$ The unsteady character of the drop heating:

Studies by Waldman (1975) [59] and Law (1976) [3] have shown that the quasi-stationary phenomenon is not verified for the liquid phase. Indeed, they observed a transient period during the heating of the drop.

$\checkmark$ Modification of the physico-chemical properties: 


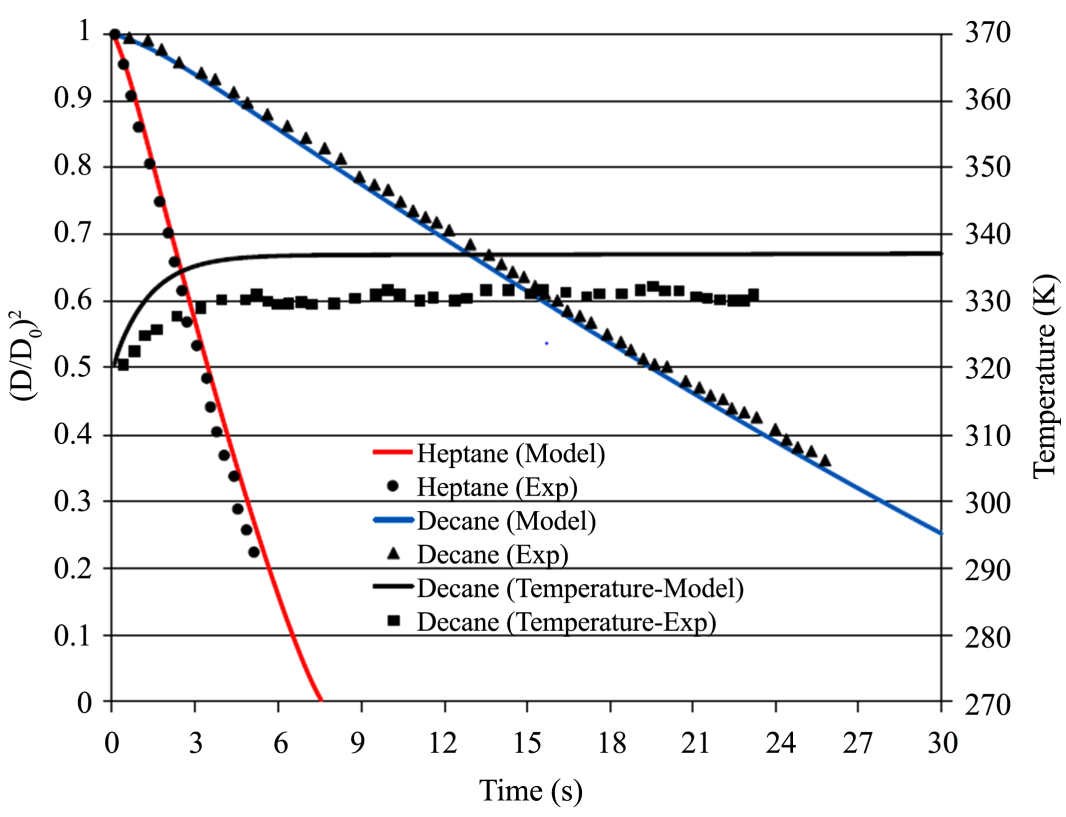

Figure 6. Comparisons between the model and the experimental data for pure heptane and decane droplet [58].

Numerical studies reported by Hubbard et al. (1975) [35] have shown the influence of the variation of physico-chemical properties on the engine performance. In 1971, Lazard and Faeth [5] showed that there was a relationship between temperature and the thermophysical properties of the liquid and vapour phases. They show that thermal conductivity and heat capacities are linearly proportional to temperature.

$\checkmark$ The influence of pressure on the thermophysical properties can create significant deviations in the quasi-stationarity of the transfers in the liquid and vapour phases.

\subsection{Liquid Phase}

\subsubsection{The Infinite Conductivity Model}

This is a model developed since 1976 by Law et al. [3]. This model is based on the " $\mathrm{d} 2$ " law which takes into account the heating of the drop. Comparative studies show that this model is similar to that of Godsave and Spalding with a slight difference related to the variation of the temperature as a function of time for that of Law et al. [3]. Also, the results of different works indicate that at high Reynolds numbers the amount of heat at the surface of the drop has an intense direct impact on the motions of the liquid phase. These movements contribute to the heating of the drop to the equilibrium temperature with the surrounding medium [12] [20] [24] [27] [29] [46] [60]. This model also relies on the evolution of temperature, drop composition and drop diameter over time. The evolution of the temperature from the heat flux $\dot{Q}_{l}$, obeys:

$$
\frac{4}{3} \pi r_{s}^{3} \rho_{l} C p_{l} \frac{\mathrm{d} T_{l}}{\mathrm{~d} t}=\dot{Q}_{l}
$$




$$
\dot{Q}_{l}=\dot{m}\left[\frac{C p_{l}\left(T_{\infty}-T_{s}\right)}{B_{T}}-L_{v}\right]
$$

The evolution of the drop diameter with time is deduced from the vapour diffusion equation by:

$$
\dot{m}=-4 \pi \rho_{l} r_{s}^{2} \frac{\mathrm{d} r_{s}}{\mathrm{~d} t}
$$

\subsubsection{Finite or Limited Conductivity Model}

This model does not take into account the motion inside the drop but rather the thermal diffusion [61]. Convection in the drop is neglected and concentration and temperature gradients are retained [44] [54] [62]. The temperature evolution is calculated from the following species and energy conservation equations:

$$
\frac{\partial T}{\partial t}=\frac{\lambda_{l}}{\rho_{l} C p_{l} r^{2}} \frac{\partial}{\partial r}\left(r^{2} \frac{\partial T}{\partial r}\right)
$$

\subsection{3. "Hill Vortex" Model}

This is a very complex model that takes into account convective transfers in the liquid and vapour phases [29] [63]. The influence of shear stresses at the liquid-vapour interface and the motions give rise to an axial velocity distribution. The expression of the velocity in polar coordinate is a function of $r$ and $\theta$ and verifies the following relations:

$$
\begin{aligned}
& V_{r}=U_{s}\left(1-\frac{r^{2}}{r_{s}^{2}}\right) \cos (\theta) \\
& V_{\theta}=U_{s}\left(1-2 \frac{r^{2}}{r_{s}^{2}}\right) \sin (\theta)
\end{aligned}
$$

With $\left\{\begin{array}{l}V_{r}: \text { radial component of velocity }\left(\mathrm{m} \cdot \mathrm{s}^{-1}\right) \\ V_{\theta}: \text { orthoradial component of the velocity }\left(\mathrm{m} \cdot \mathrm{s}^{-1}\right) . \\ U_{s}: \text { surface velocity of the liquid }\left(\mathrm{m} \cdot \mathrm{s}^{-1}\right)\end{array}\right.$

In this model only the internal convection related to the relative velocity with respect to the gas phase is taken into account. Indeed, the effects of additional surface stresses related to temperature or composition are neglected by the assumption of uniformity of temperature and composition at the surface of the drop.

None of the above models with the mentioned equations take into account the contribution of advection or azimuthal angle, which are present in convective evaporation. This should be taken into account in order to bring out all aspects of the evaporation and combustion of liquid fuel drops.

\section{Experimental Study: Influence of Temperature and Pressure}

The study of the evaporation of one or more drops composed of one or more 
components has been the subject of several experimental works [29] [46] [49] in recent years. This work has also focused on the effects of temperature, pressure and convection on the evaporation and combustion of one or more drops consisting of one or more components.

X. Chesneau (1994) [29] carried out an experimental and numerical study on the natural convection evaporation of a drop under the influence of vapour pressure. Indeed, gravity induces buoyancy forces on the drop causing its deformation. Also, by the critical pressure and for a temperature close to the critical temperature cancel the surface tension and the latent heat of vaporization of the drop.

Kadota and Hiroyasu [64] presented experimental results of the evaporation of a drop of $n$-heptane in nitrogen under high pressure and established a numerical correlation similar to that of Ranz and Marshall for the Nusselt and Sherwood numbers.

C. Chauveau [30] was interested in experimental work on the evaporation and combustion of a drop in microgravity using the free fall technique. He visualized with a CCD camera the evolution of the radius of drops at high and low pressure.

A. S. Zongo [52] showed experimentally that for a temperature between $350^{\circ} \mathrm{C}$ and $440^{\circ} \mathrm{C}$, there is degradation of the monitored drop. He shows that the evolution of the drop as a function of temperature can contribute to the prediction of its evaporation.

\section{Combustion of Fuel Drops}

\subsection{Numerical Synthesis}

The modelling of the combustion of one or more spherical drops has been the subject of several works [3] [18] [28] [53] [65] [66]. Among these works, the simplified Godsave and Spalding model has been widely used. Some works have focused on the analysis of the combustion of an isolated spherical droplet without in the liquid phase [63] [67]. Other works are based on the coupling between mass and heat transfer in the liquid and vapour phases in the absence or presence of gravity [68] [69].

\subsection{Experimental Study}

The experimental study of the combustion of a drop dates back many years. Already in 1971 Kumasi et al. visualized the regression of the diameter of a drop in microgravity using a CCD camera.

M. El-Kasaby et al. [70] carried out experimental work on the performance and ignition delay of an engine running on jatropha biodiesel with convincing results.

D QI et al. [71] presented their experimental results on the emissions from the combustion of the oil-diesel mixture in the engine and found a clear decrease in greenhouse gases. Some of the results of the work have been presented in Table 2. 


\section{Synthesis}

Zainab Abdel-Qadera and William L. H. Hallett, 2005 [53] investigated the role of liquid mixing in the evaporation of multicomponent mixtures through continuous thermodynamics modelling. The aim was to understand the role played by internal mixing in the evaporation of droplets from mixtures with a large number of components. The calculations show that internal mixing generally has a smaller influence on droplet behaviour for a mixture with a large number of components than for a binary mixture and give some indication of when a well-mixed droplet model may-or may not-be a good approximation for practical work.

B. Abramzon et al., 2006 [54] were interested in the convective vaporization of a fuel droplet with radiation absorption. The developed "extended" and "effective conductivity" droplet vaporization models are generalized to take into account the contribution of thermal radiation and humidity and the temperature dependence of the liquid fuel properties. Therefore, they simulate the effect of thermal radiation and absorption of thermal radiation in a semi-transparent spherical droplet using the simplified thermal radiation absorption model proposed by Dombrovsky and Sazhin: a simplified model. The physical properties of the liquid fuel, including density, are evaluated at the average temperature of the liquid and updated at each time step. These generalized models are applied to the analysis of the vaporization process of $\mathrm{n}$-decane and diesel fuel droplets injected into hot air. It is shown that the absorption of radiation in diesel fuel is generally stronger than in n-decane, and this must be taken into account when modelling the combustion processes in diesel engines. Calculations of the droplet vaporization rate using the simplified "effective conductivity" model with the uniform internal radiation heat source. This allows us to recommend the use of the "effective conductivity" model with uniform radiation absorption for vaporization combustion calculations, including applications in internal combustion engines.

J. Barata, 2008 [55] have modelled the dispersion and evaporation of biofuel droplets. This paper presents a numerical study of the evaporation of biofuel droplets injected into a turbulent crossflow. This study uses an Eulerian/Lagragian approach to account for turbulent transport, dispersion, evaporation and the coupling between the two processes in practical spray injection systems, which typically involve two-way air flows. A set of biofuel droplets evaporating through a crossflow is studied, and a comparison of the dispersion and evaporation of the droplet fuel with conventional fuels is made. Droplet evaporation with conventional fuels. The results obtained with DF-2 and RME showed that a homogeneous mixture can only be obtained with very high levels of preheating, and that the use of ethanol (obtained from sugar or starch) can be a better alternative in ignition assisted combustion systems, while RME can be successfully used as an alternative fuel in applications that use diffusion flames. An Eulerian/Lagragian approach was presented to calculate evaporative sprays through a cross-flow. A 
method developed to study two- and three-dimensional turbulent isothermal dispersion was extended to the case of an evaporating biofuel droplet array. The results show that the distributions of droplet diameter, temperature and mass fraction were found to be strongly dependent on the fuels used. Rapeseed methyl esters show similar evaporation characteristics to DF-2, indicating that they can be successfully used as an alternative fuel in many applications.

Zhou Zhifu et al. 2013 [56] evaluated evaporation models for a single moving drop with a high evaporation rate and conducted experiments of a single evaporating drop subjected to a gas flow. The objective of their work was to evaluate existing evaporation models with a high evaporation rate. From the results obtained, it appears that all models perform almost identically for cases with low evaporation rates, while significant discrepancies between the models' predictions appear when the evaporation rate is increased. Based on these comparisons, a simplified evaporation model is identified and recommended for high evaporation cases. The comparison also reveals a lack of reliable experimental temperature data for drop evaporation, especially for high evaporation conditions. Thus, attention is given to three phenomena of droplet evaporation: Stefan flow, convective heat and mass transfer, and the blowing effect due to high evaporation rate. In particular, various methods for modelling convective heat and mass transfer with a high blow-off effect are examined theoretically and experimentally. The validity of various models for droplet evaporation is tested by comparing model predictions with three sets of experimental data with low, intermediate and high evaporation rates, respectively. It is found that all models perform almost identically for the low evaporation cases while significant large discrepancies between the model predictions emerge as the evaporation rate increases.

J. Dgheim et al., 2013 [14] conducted a numerical study of heat and mass transfer from a hydrocarbon droplet rotating around its vertical axis in forced convection only on the opposite side of the flow. The flow was assumed to be laminar, and the droplet was assumed to retain its spherical shape during its lifetime. Based on the above assumption, the conservation equations in a general curvilinear coordinate were solved numerically. The evaporation behaviour of rotating droplets in a forced convection flow can be studied by analyzing the effects of droplet rotation on the evaporation process of a multicomponent hydrocarbon droplet. The droplet is simulated to behave as a hard sphere. The thickness of the boundary layer is unknown for this model and therefore it was determined as a function of time. In addition, the study focuses on the Dgheim dimensionless number which is the ratio of the rotational forces to the viscosity forces. The Dgheim dimensionless number is correlated to the Nusselt and Sherwood numbers for multicomponent hydrocarbon droplets in evaporation taking into account the effect of heat and mass of the Spalding, Prandtl and Schmidt numbers respectively. Similarly, correlations for the Nusselt and Sherwood numbers as a function of the Reynolds, Prandtl and Schmidt numbers are proposed. These 
correlations take into account the rotation phenomenon and advance the variation of thermophysical and transport properties in the vapour phase of multi-component mixtures.

Tomoaki Kitano et al., 2014 [57] investigated the effects of ambient pressure, gas temperature and combustion reaction on droplet evaporation using a threedimensional numerical simulation. The ambient pressure, initial gas temperature and droplet mass loading ratio are varied in the ranges of $0.1-2.0 \mathrm{MPa}$, $1000-2000 \mathrm{~K}$ and $0.027-0.36$, respectively, under conditions with and without combustion reaction. The results show that for both conditions with and without combustion reaction, the droplet lifetime increases with the combustion reaction, with the increase of ambient pressure at a low initial gas temperature of $1000 \mathrm{~K}$, but decreases at the high initial temperatures of $1500 \mathrm{~K}$ and $2000 \mathrm{~K}$, although the droplet lifetime is shorter due to the combustion reaction. Also, it is noted that the increase in ambient pressure and the inhomogeneity of the droplet distribution due to turbulence cause the droplet lifetime to be generally shorter. The main results obtained in their paper show that:

1) Natural convection accelerates droplet evaporation, and the effect becomes noticeable with increasing ambient temperature.

2) In the condition without combustion reaction, the droplet lifetime increases with increasing ambient pressure at low initial gas temperature of $1000 \mathrm{~K}$, but decreases at higher initial temperature. This is attributed to an equilibrium between increasing and decreasing droplets. Indeed, as the ambient pressure increases, droplet evaporation is suppressed by a higher boiling temperature, while it is favored by a lower latent and a larger droplet surface area due to a larger liquid expansion.

3) The effects of ambient pressure, initial gas temperature and ML on droplet lifetime in the combustion reaction condition are similar to those in the no combustion reaction condition.

However, the droplet lifetime is often shortened by an improved combustion reaction in the following conditions of high ambient pressure and moderately high ML.

4) Both for the conditions with and without combustion reaction, the inhomogeneity of droplet distribution caused by turbulence makes the droplet lifetime longer because the local ML increases considerably at some locations.

M. Al Qubeissi et al. 2015 [16] were interested in modelling the heating and evaporation of biodiesel fuel droplets. To do so, they made a comparative analysis of the predictions of several biodiesel droplet heating and evaporation models under realistic diesel engine conditions. Subsequently, for the analysis of their work, they used nineteen types of biodiesel fuels composed of methyl esters. It is shown that the model, based on the assumption that the diffusivity of the species in the droplets is infinitely fast and the thermal conductivity of the liquid is infinitely large, under predicts the evaporation time of the droplets compared to the model taking into account the effects of finite diffusivity and conductivity, by up to about $15 \%$. A similar under-prediction of the model in which transient spe- 
cies diffusion is ignored and the thermal conductivity of the liquid is assumed to be infinitely large, turns out to be about $26 \%$. As in the case of diesel and gasoline droplets, for biodiesel droplets, the multi-component models predict higher droplet surface temperatures at the final stages of droplet evaporation and longer evaporation times than for the single-component models. This is related to the fact that in the final stages of droplet evaporation, the mass fraction of heavier species, which evaporate more slowly than lighter species and have higher boiling temperatures, increases at the expense of lighter species.

Xiaokang Ma 2016 [58] numerically modelled the evaporation process of droplets from acetone-butanol-ethanol $(\mathrm{ABE})$ and diesel mixtures. The evaporation characteristics of acetone-butanol-ethanol (ABE) and diesel mixtures are investigated using a multi-component evaporation model coupled with six components to represent diesel and the universal functional activity coefficient (UNIFAC) method was constructed and validated. The evaporation characteristics of the $\mathrm{ABE}$-mixture as well as the effects of $\mathrm{ABE}$ mass fraction and ambient temperature are analyzed.

The results show that droplets of the $\mathrm{ABE}$-diesel mixture evaporate faster than diesel. The addition of $\mathrm{ABE}$ affects the early stage of the evaporation process, especially at high temperatures, which leads to the appearance of internal gasification observed in the experiments. As the mass fraction of $\mathrm{ABE}$ increases, the evaporation rate increases and internal gasification is more likely to occur later, but the preform is more intense. Droplet lifetime decreases non-linearly with increasing ambient temperature, and evaporation of low volatile components is significantly improved. At the same time, the increase in ambient temperature leads to faster and more intense internal gasification performance.

M. Al Qubeissi et al., 2017 [59], in his work thought about the modelling of heating and evaporation of droplets of a diesel-biodiesel mixture. He presents a new approach to modelling the heating and evaporation of biodiesel droplets with specific application to blends of biodiesel (represented by the widely used soybean methyl ester, SME) and diesel fuels under conditions representative of internal combustion engines. The compositions, approximately 105 components of diesel and biodiesel fuels, are replaced by a smaller number of components and quasi-components using the compositional analysis method. The results are compared with predictions for the case where the biodiesel/diesel blend droplets are represented by pure biodiesel or pure diesel droplets. In their work it is shown that the predicted droplet evaporation time and surface temperature for $100 \%$ SME, representing pure biodiesel, are close to those predicted for pure diesel. It is also shown that approximations of the actual compositions of B5 (5\% SME and 95\% Diesel) and B50 (50\% SME and 50\% Diesel) by 17 quasi-components/components, using the MDQD model, lead to under-predictions of droplet lifetime of up to $9 \%$ and $4 \%$, respectively, under the same engine conditions. The application of the latter model resulted in a reduction of more than $83 \%$ in computation time compared to the case where 105 components are used.

Fang Wang et al., 2017 [9] proposed a new stationary drop evaporation model 
and its validation. They showed the importance of the character of liquid drop evaporation not only for the design process of the combustion chamber, but also for the simulation of high accuracy spray combustion. In his work, the evaporation character of suspended drops was measured in a quiet environment at high temperature by a high-speed micro camera system. The experimental results for gasoline and paraffin are consistent with the reference data. The experimental evaporation rate data were compared with the prediction results of the RanzMarshall boiling temperature model (RMB), the Ranz-Marshall low temperature model (RML), the drift flow model (DFM), the mass analogy and the temperature analogy. The discrepancy between the experimental data and the model prediction results was mainly due to the neglect of the natural convection effect, which was never introduced in the model. A new drop evaporation model taking into account the buoyancy effect of natural convection was also proposed. The results show that

1) The evaporation characteristics of liquid fuel generally correspond to the $\mathrm{d} 2$ law and the evaporation rate increases with increasing ambient temperature.

2) Calculations of the Ranz-Marshall evaporation model and other models that do not consider natural convection deviate significantly from experimental results.

3) The computational results of the new droplet evaporation model are consistent with the experimental data for room temperature.

4) The new droplet evaporation model that takes into account natural convection can predict droplet evaporation more accurately than conventional models, such as the boiling evaporation model of Ranz and Marshall. Marshall boiling evaporation model.

Dgheim et al., 2018 [60] investigated the combustion of droplets in rotating natural convection. This study, based on experimental and numerical, provided a thermal and fluid flow analysis of the evaporation and combustion of liquid hydrocarbon droplets in natural and rotating convection. The results obtained allowed the determination of the regression of the square of the liquid droplet diameter, the evolution of the surface temperature, the variation of the temperature and velocity in the liquid and vapour phases, and the evolution of the radius and temperature of the flame in natural rotating convection. The comparison between the numerical and experimental results shows a satisfactory qualitative and quantitative agreement. In addition, a correlation expressing the burning rate as a function of the Dgheim, Prandtl and Grashof numbers is proposed. The burning rate results from the burning rate without convection, the burning rate due to stagnant natural convection and the burning rate due to rotating natural convection.

Hyemin Kim 2018 [61] carried out work on the evaporation of a single droplet of emulsion fuel under high temperature and pressure conditions. The evaporation characteristics of water/n-decane emulsion droplets at various temperatures and pressures were observed experimentally. The emulsion fuel was made by adding pure water to the $\mathrm{n}$-decane base fuel with a volume ratio of 0.2:0.2. The 
time variation of the droplet diameter was observed optically with a high speed camera. The evaporation process of the emulsion droplets was divided into three stages, namely drop heating, swelling/blowing and pure evaporation. As the ambient temperature increased, the droplet swelling behaviour shifted towards swelling during the swelling/blowing phase. There was a decrease in the inflation/blowing rate under high pressure conditions. The evaporation rate during the pure evaporation stage and the overall droplet lifetime were affected by ambient temperature but not by ambient pressure. Droplet swelling occurred mainly at relatively low temperature and pressure conditions; it transformed into blowing at higher temperature and pressure conditions.

Dgheim 2020 [11] conducted a new study of the evaporation of multicomponent hydrocarbon liquid droplets in rotating forced convection. For this purpose, they numerically investigated the heat and mass transfer of evaporation of ternary-component liquid hydrocarbon droplets in rotary forced convection. Optimal values for the wind and rotation velocities of the liquid droplet were determined. Further new correlations expressing the evaporation rate in terms of Dgheim, Reynolds, Prandtl and Schmidt numbers are made which take into account the evaporation of the ternary component liquid droplet at varying ambient temperatures, varying mass fractions, varying air velocities and varying initial droplet radii, under forced rotating convection.

\section{Conclusion}

The state of the art has shown the different phenomena related to the evaporation of a drop of liquid fuel, the modes of transfer as well as the parameters influencing the evaporation. It also recalled the different models used in numerical work on the evaporation and combustion of one or more drops of fuel. There are very few studies on the influence of natural convection as opposed to forced convection, especially on an engine fuelled by biodiesel. The experimental techniques, for the most part, use thermocouples to measure the temperature of the evaporating drop surface and the CCD camera to visualize the regression of the diameter squared of the drop.

\section{Conflicts of Interest}

The authors declare no conflicts of interest regarding the publication of this paper.

\section{References}

[1] Faeth, G.M. (1983) Evaporation and Combustion of Sprays. Progress in Energy and Combustion Science, 9, 1-76. https://doi.org/10.1016/0360-1285(83)90005-9

[2] Sirignano, W.A. (1983) Fuel Droplet Vaporization and Spray Combustion Theory. Progress in Energy and Combustion Science, 9, 291-322. https://doi.org/10.1016/0360-1285(83)90011-4

[3] Law, C. (1982) Recent Advances in Droplet Vaporization and Combustion. Prog Energy Combust, 8, 171-201. https://doi.org/10.1016/0360-1285(82)90011-9 
[4] Dgheim, J. (2001) Contribution à l'étude de l'évaporation et de la combustion de gouttes de carburants liquides. Université de Perpignan Via Domitia, Perpignan.

[5] Lazard, R.S. and Faeth, G. (1971) Bipropellant Droplet Combustion in the Vicinity of the Critical Point. Symposium (International) on Combustion, 13, 801-811. https://doi.org/10.1016/S0082-0784(71)80082-6

[6] Faeth, G. (1977) Current Status of Droplet and Liquid Combustion. Progress in Energy and Combustion Science, 3, 191-224. https://doi.org/10.1016/0360-1285(77)90012-0

[7] Mauduit, J. (1992) Contribution à l'étude de la vaporisation et de la combustion de gouttes isolées, introduction aux effets des hautes pressions. Université d'Orléans, Orléans.

[8] Abramzon, B. and Sirignano, W.A. (1989) Droplet Vaporization Model for Spray Combustion Calculations. International Journal of Heat and Mass Transfer, 32, 1605 1618. https://doi.org/10.1016/0017-9310(89)90043-4

[9] Bencherif, M. (2014) Contribution à l'étude des interactions combustion turbulence dans les moteurs à combustion interne. Université des Sciences et de la Technologie d'Oran “Mohamed Boudiaf”, Bir El Djir.

[10] Merouane, H. (2013) Étude de la vaporisation des gouttes de combustibles liquides en écoulement sous effet thermique. Université des Sciences et de la Technologie d'Oran “Mohamed Boudiaf”, Bir El Djir.

[11] Yuge, T. (1960) Experiment on Heat Transfer from Spheres Including Natural and Forced Convection. Journal of Heat Transfer, 82, 214-220. https://doi.org/10.1115/1.3679912

[12] Doué, N. (2005) Modélisation de l'évaporation de gouttes multi-composants. École nationale supérieure de l'aéronautique et de l'espace, Toulouse,

[13] Khiari, K. (2016) Contribution à l'étude des propriétés thermo-physiques des biocarburants de seconde génération et leur influence sur le comportement des moteurs. Ecole des Mines de Nantes, Nantes.

[14] Haywood, R., Nafziger, R. and Renksizbulut, M. (1989) A Detailed Examination of Gas and Liquid Phase Transient Processes in Convective Droplet Evaporation. Journal of Heat Transfer, 111, 495-502. https://doi.org/10.1115/1.3250704

[15] Nje-Nje, C. (2000) Etude numérique de l'évaporation instationnaire d'un nuage de gouttes multicomposants. Université de ROUEN, Mont-Saint-Aignan.

[16] Daho, T. (2008) Contribution à l'étude des conditions optimales de combustion des huiles végétales dans les moteurs diesel et sur les brûleurs: Cas de l'huile de coton. Université de Ouagadougou, Ouagadougou.

[17] Wang, F., Yao, J., Yang, S., Liu, R. and Jin, J. (2017) A New Stationary Droplet Evaporation Model and Its Validation. Chinese Journal of Aeronautics, 30, 14071416. https://doi.org/10.1016/j.cja.2017.06.012

[18] Sazhin, S.S. (2017) Modelling of Fuel Droplet Heating and Evaporation: Recent Results and Unsolved Problems. Fuel, 196, 69-101. https://doi.org/10.1016/j.fuel.2017.01.048

[19] Dgheim, J., Al Maarrawi, R., Abdallah, M. and Nasr, N. (2020) New Investigation of the Evaporation of Multicomponent Hydrocarbons Liquid Droplets in Rotatory Forced Convection.

[20] Daif, A., Bouaziz, M., Bresson, J. and Grisenti, M. (1999) Surface Temperature of Hydrocarbon Droplet in Evaporation. Journal of Thermophysics and Heat Transfer, 13, 553-556. https://doi.org/10.2514/2.6479 
[21] Bouaziz, M., Grisenti, M., Dedies, R. and Zeghmati, B. (2002) Heat and Mass Transfer Correlation for a Porous Sphere Saturated with Liquid Evaporated in Natural Convection Flow. Chemical Engineering and Processing. Process Intensification, 41, 539-549. https://doi.org/10.1016/S0255-2701(01)00174-X

[22] Dgheim, J. and Zeghmati, B. (2005) Heat and Mass Transfer Investigation of Hydrocarbon Droplet Evaporation under Rotatory Movement. Chinese Physics Letters, 22, 2933-2935. https://doi.org/10.1088/0256-307X/22/11/057

[23] Sazhin, S.S., Qubeissi, M.A., Kolodnytska, R., Elwardany, A.E., Nasiri, R. and Heikal, M.R. (2013) Modelling of Biodiesel Fuel Droplet Heating and Evaporation. International Heat Transfer Conference 15, Kyoto, 10-15 August 2014, 2403-2414. https://doi.org/10.1615/IHTC15.evp.008936

[24] Dgheim, J., Chesneau, X., Pietri, L. and Zeghmati, B. (2001) Etude numérique des transferts lors de la combustion en convection naturelle d'une goutte de carburant liquide.

[25] Dgheim, J., Abdallah, M. and Nasr, N. (2017) Enhanced Evaporation of Droplet of Ternary Component under the Effect of Thermo-Physical and Transport Properties Variability. Arabian Journal for Science and Engineering, 43, 2181-2194. https://doi.org/10.1007/s13369-017-2561-8

[26] Daho, T., Vaitilingom, G., Sanogo, O., Ouiminga, S.K., Segda, B.G., Valette, J., et al. (2012) Model for Predicting Evaporation Characteristics of Vegetable Oils Droplets Based on Their Fatty Acid Composition. International Journal of Heat and Mass Transfer, 55, 2864-2871. https://doi.org/10.1016/j.ijheatmasstransfer.2012.01.048

[27] Dgheim, J., Abdallah, M., Habchi, R. and Zakhia, N. (2012) Heat and Mass Transfer Investigation of Rotating Hydrocarbons Droplet Which Behaves as a Hard Sphere. Applied Mathematical Modelling, 36, 2935-2946. https://doi.org/10.1016/j.apm.2011.09.078

[28] Sazhin, S.S., Al Qubeissi, M. and Xie, J.F. (2014) Two Approaches to Modelling the Heating of Evaporating Droplets. International Communications in Heat and Mass Transfer, 57, 353-356. https://doi.org/10.1016/j.icheatmasstransfer.2014.08.004

[29] Chesneau, X. (1994) Vaporisation et combustion de gouttes isolées de combustibles liquides. Influence de la pression. Thèse de Doctorat, Université d'Orléans, Orléans.

[30] Chauveau, C., Chesneau, X. and Gijkalp, I. (1995) High Pressure Vaporization and Burning of Methanol Droplets in Reduced Gravity. Advances in Space Research, 16, 157-160. https://doi.org/10.1016/0273-1177(95)00152-5

[31] Chahine, A.J. (2017) Contribution à l'étude numérique et expérimentale de l'évaporation and la combustion des gouttes d'hydrocarbures liquides en rotation. Université Libanaise, Beirut.

[32] Dabilgou, T., Dgheim, J., Chesneau, X. and Koulidiati, J. (2021) Evaporation Improvement in Natural Convection of Heptane Liquid Droplet. International Journal of Current Research, 13, 16084-16101.

[33] Qubeissi, M.A., Sazhin, S.S., Crua, C., Turner, J. and Heikal, M.R. (2015) Modelling of Biodiesel Fuel Droplet Heating and Evaporation: Effects of Fuel Composition. Fuel, 154, 308-318. https://doi.org/10.1016/j.fuel.2015.03.051

[34] Habibullah, M., Masjuki, H.H., Kalam, M.A., Rizwanul Fattah, I.M., Ashraful, A.M. and Mobarak, H.M. (2014) Biodiesel Production and Performance evaluation of Coconut, Palm and Their Combined Blend with Diesel in a Single-Cylinder Diesel Engine, 87, 250-257. https://doi.org/10.1016/j.enconman.2014.07.006

[35] Hubbard, G.L., Denny, V.E. and Mills, A.F. (1975) Droplet Evaporation: Effects of Transients and Variable Properties. International Journal of Heat and Mass Transfer, 
18, 1003-1008. https://doi.org/10.1016/0017-9310(75)90217-3

[36] Tonini, S. and Cossali, G. (2012) An Analytical Model of Liquid Drop Evaporation in Gaseous Environment. International Journal of Thermal Sciences, 57, 45-53. https://doi.org/10.1016/j.ijthermalsci.2012.01.017

[37] Maqua, C., Castanet, G. and Lemoine, F. (2008) Bicomponent Droplets Evaporation: Temperature Measurements and Modelling. Fuel, 87, 2932-2942. https://doi.org/10.1016/j.fuel.2008.04.021

[38] Abramzon, B. and Sazhin, S.S. (2006) Convective Vaporization of a Fuel Droplet with Thermal Radiation Absorption. Fuel, 85, 32-46. https://doi.org/10.1016/j.fuel.2005.02.027

[39] Kitano, T., Nishio, J., Kurose, R. and Komori, S. (2014) Effects of Ambient Pressure, Gas Temperature and Combustion Reaction on Droplet Evaporation. Combustion and Flame, 161, 551-564. https://doi.org/10.1016/j.combustflame.2013.09.009

[40] Maqua, C., Castanet, G., Grisch, F., Lemoine, F., Kristyadi, T. and Sazhin, S.S. (2008) Monodisperse Droplet Heating and Evaporation: Experimental Study and Modelling. International Journal of Heat and Mass Transfer, 51, 3932-3945. https://doi.org/10.1016/j.ijheatmasstransfer.2007.12.011

[41] Dgheim, J., Chahine, A. and Nahed, J. (2018) Investigation on the Droplet Combustion in Rotatory Natural Convection. Journal of King Saud University. Science, 31, 937-945. https://doi.org/10.1016/j.jksus.2018.02.007

[42] Merouane, H. and Bounif, A. (2010) Theoretical and Numerical Analysis of Fuel Droplet Vaporisation at high Temperatures. WSEAS Transactions on Heat and Mass Transfer, 5, 189-196.

[43] Pinheiro, A.P., Vedovoto, J.M., da Silveira Neto, A. and van Wachem, B.G. (2019) Ethanol Droplet Evaporation: Effects of Ambient Temperature, Pressure and Fuel Vapor Concentration. International Journal of Heat and Mass Transfer, 143, Article ID: 118472. https://doi.org/10.1016/j.ijheatmasstransfer.2019.118472

[44] Raghavan, V. (2019) Numerical Modeling of Evaporation and Combustion of Isolated Liquid Fuel Droplets: a Review. Journal of the Indian Institute of Science, 99, 5-23. https://doi.org/10.1007/s41745-019-0097-5

[45] Belkhelfa, Y. (2008) Étude du comportement dynamique et du transfert de matière et de chaleur entre des parti-cules sphériques et un écoulement laminaire ou turbulent. Institut national des sciences appliquees de rouen, Saint-Étienne-du-Rouvray.

[46] Bouaziz, M., Dgheim, J., Grisenti, M., Bresson, J. and Zeghmati, B. (2002) Experimental and Numerical Study in Evaporation of Hydrocarbon Droplet Surface Temperature. $A R I, 53,16-24$.

[47] Renksizbulut, M. and Yuen, M.C. (1983) Numerical Study of Droplet Evaporation in a High-Temperature Stream. Journal of Heat Transfer, 105, 389-397. https://doi.org/10.1115/1.3245591

[48] Scherer, G.W. (1987) Drying Gels III. Warping Plate. Journal of Non-Crystalline Solids, 91, 83-100. https://doi.org/10.1016/S0022-3093(87)80087-X

[49] Dgheim, J., Chesneau, X., Pietri, L. and Zeghmati, B. (2002) Heat and Mass Transfer Correlations for Liquid Droplet of a Pure Fuel in Combustion. Heat and Mass Transfer, 30, 543-550. https://doi.org/10.1007/s002310100202

[50] Ranz, W.E. and Marshall, W.R. (1952) Evaporation from Drops. Chemical Engineering Progress, 48, 141-146.

[51] Sazhin, S.S. (2018) Modelling of Droplet Heating and Evaporation. In: Basu, S., Agarwal, A., Mukhopadhyay, A. and Patel, C., Eds., Droplets and Sprays, Springer, 
Singapore, 45-75. https://doi.org/10.1007/978-981-10-7449-3 3

[52] Zongo, S.A. (2015) Étude des processus physiques et chimiques mis en jeu lors de la combustion des huiles végétales pures dans les moteurs diesel: Mécanismes de décomposition et de polymérisation. Université de Ouagadougou, Ouagadougou.

[53] Demirbas, A. (2008) Mathematical Relationships Derived from Biodiesel Fuels. Energy Sources, Part A: Recovery, Utilization, and Environmental Effects, 30, 56-69. https://doi.org/10.1080/00908310600626762

[54] Godsave, G.A.E. (1953) Studies of the Combustion of Drops in a Fuel Spray: The Burning of Single Drops of Fuel. Symposium (International) on Combustion, 4, 818-830. https://doi.org/10.1016/S0082-0784(53)80107-4

[55] Spalding, D.B (1953) The Combustion of Liquid Fuels. Symposium (International) on Combustion, 4, 847-864. https://doi.org/10.1016/S0082-0784(53)80110-4

[56] Williams, F.A. (1973) Combustion of Droplets of Liquid Fuels. Combustion and Flame, 21, 1-31. https://doi.org/10.1016/0010-2180(73)90002-3

[57] Sparrow, E.M. and Gregg, J.L. (1958) The Variable Fluid-Property Problem in Free Convection. Transactions of ASME, 80, 879-886. https://doi.org/10.1115/1.4012546

[58] Gu, X. (2012) Numerical Simulation of Conventional Fuels and Biofuels Dispersion and Vaporization Process in Co-Flow and Cross-Flow Premixers. Electronic Theses and Dissertations, No. 2314. http://stars.library.ucf.edu/etd/2314

[59] Waldman, C.H. (1975) Theory of Non-Steady State Droplet Combustion. Symposium (International) on Combustion, 15, 429-442. https://doi.org/10.1016/S0082-0784(75)80317-1

[60] Strotos, G., Gavaises, M., Theodorakakos, A. and Bergeles, G. (2011) Numerical Investigation of the Evaporation of Two-Component Droplets. Fuel, 90, 1492-1507. https://doi.org/10.1016/j.fuel.2011.01.017

[61] Bouaziz, M. (1998) Etude numérique et expérimentale de l'évaporation simultanée de plusieurs gouttes de carburants: Influence de la pression, des convections et e l'interaction mécanique. Perpignan Via Domitia, Perpignan.

[62] Knothe, G. and Razon, L.F. (2017) Biodiesel Fuels. Progress in Energy and Combustion Science, 58, 36-59. https://doi.org/10.1016/j.pecs.2016.08.001

[63] Cho, S.Y. and Dryer, F.L. (1999) A numérical Study of the Unteady Burning Behavior of N-Heptane Droplets. Combustion Theory and Modelling, 3, 267-280. https://doi.org/10.1088/1364-7830/3/2/004

[64] Kadota, T. and Hiroyasu, H. (1976) Evaporation of a Single Droplet at Elevated Pressures and Temperatures. Bulletin of the JSME, 19, 1515-1521. https://doi.org/10.1299/jsme1958.19.1515

[65] Rajasekar, E. and Selvi, S. (2014) Review of Combustion Characteristics of CI Engines Fueled with Biodiesel. Renewable and Sustainable Energy Reviews, 35, 390-399. https://doi.org/10.1016/j.rser.2014.04.006

[66] Rah, S.C., Sarafim, A.F. and Beer, J.M. (1986) Ignition and Combustion of Liquid Fuel Droplets: Part I. Impact on Pollution Formation. Combustion Science and Technology, 48, 273-284. https://doi.org/10.1080/00102208608923897

[67] Marchese, A.J. and Dryer, F.L. (1996) The Effect of Liquid Mass Transport on the Combustion and Extinction of Bicomponent Dropets of Methanol and Water. Combustion and Flame, 105, 104-122. https://doi.org/10.1016/0010-2180(95)00179-4

[68] Shaygan, N. and Prakash, S. (1995) Droplet Ignition and Combustion Including Liquid-Phase Heating. Combustion and Flame, 102, 1-10. 
https://doi.org/10.1016/0010-2180(95)00027-4

[69] Cho, S.Y., Yetter, R.A. and Dryer, F.L. (1992) Computer Model for Chemically Reacting Flow with Complex Chemistry, Multi-Component Diffusion and Heterogenous Processes. Journal of Computational Physics, 102, 432-459. https://doi.org/10.1016/S0021-9991(05)80013-0

[70] El-Kasaby, M. and Nemit-Allah, M. (2013) Experimental Investigations of Ignition Delay Period and Performance of a Diesel Engine Operated with Jatropha Oil Biodiesel. Alexandria Engineering Journal, 52, 141-149.

https://doi.org/10.1016/j.aej.2012.12.006

[71] Qi, D., Lee, C., Jia, C., Wang, P. and Wu, S. (2014) Experimental Investigations of Combustion and Emission Characteristics of Rapeseed Oil-Diesel Blends in a TwoCylinder Agricultural Diesel Engine. Energy Conversion and Management, 77, 227232. https://doi.org/10.1016/j.enconman.2013.09.023 